\title{
SCIENTIFIC REPORTS

\section{OPEN Widespread Sexual Dimorphism in the Transcriptome of Human Airway Epithelium in Response to Smoking}

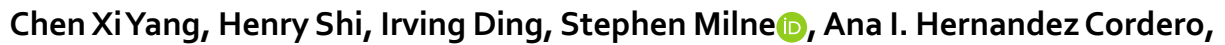 \\ Cheng Wei Tony Yang $\mathbb{D}$, Edward Kyoo-Hoon Kim, Tillie-Louise Hackett, Janice Leung, \\ Don D. Sin (1) \& Ma'en Obeidat $\mathbb{D}^{*}$
}

Epidemiological studies have shown that female smokers are at higher risk of chronic obstructive pulmonary disease (COPD). Female patients have worse symptoms and health status and increased risk of exacerbations. We determined the differences in the transcriptome of the airway epithelium between males and females, as well the sex-by-smoking interaction. We processed public gene expression data of human airway epithelium into a discovery cohort of 211 subjects (never smokers $\mathrm{n}=68$; current smokers $\mathrm{n}=143$ ) and two replication cohorts of 104 subjects ( 21 never, 52 current, and 31 former smokers) and 238 subjects (99 current and 139 former smokers. We analyzed gene differential expression with smoking status, sex, and smoking-by-sex interaction and used network approaches for modules' level analyses. We identified and replicated two differentially expressed modules between the sexes in response to smoking with genes located throughout the autosomes and not restricted to sex chromosomes. The two modules were enriched in autophagy (up-regulated in female smokers) and response to virus and type 1 interferon signaling pathways which were down-regulated in female smokers compared to males. The results offer insights into the molecular mechanisms of the sexually dimorphic effect of smoking, potentially enabling a precision medicine approach to smoking related lung diseases.

Chronic obstructive pulmonary disease (COPD) affects more than 300 million people and is the $3^{\text {rd }}$ leading cause of death worldwide ${ }^{1}$. In Canada, COPD is the leading cause of hospitalization, accounting for 80,000 hospital admissions per year ${ }^{2}$. While historically men used to smoke more, there has been a marked rise in the prevalence of smoking among women since the 1960's, leading to a sharp increase in the burden of COPD among women. In the year 2000 the number of women dying of COPD surpassed that of men in the United States ${ }^{3}$, and in Canada recent estimates suggest that there are approximately $85 \%$ more female COPD patients than male patients ${ }^{4}$. Our group has reported previously that female smokers experience a heightened risk of COPD, particularly following menopause ${ }^{5}$. These data have been validated in a study involving 149,075 women and 100,252 men in the UK Biobank $^{6}$. For the same severity of airflow obstruction, female patients have increased shortness of breath, poorer quality of life and health status, and greater functional impairment than male patients ${ }^{7}$. Furthermore, women with COPD are more likely to experience increased risk of exacerbations ${ }^{7}$.

There is a growing body of evidence from histological, imaging and animal models to support the notion that females have more airways disease and less emphysema compared to male COPD patients ${ }^{8,9}$. In a murine model where male and female (ovariectomized and ovary-intact) mice were exposed to smoke for 6 months, the female mice with intact ovaries had significantly thicker small airway walls but had similar degrees of emphysema compared with male or ovariectomized female mice ${ }^{10}$.

Females are also thought to have augmented xenobiotic metabolism of inhaled chemicals such as nicotine ${ }^{11}$. Since some metabolites of nicotine may be toxic, the sex-related differences in xenometabolism maybe an important driver of increased female susceptibility to $\mathrm{COPD}^{12,13}$. In addition, we have shown in vitro that females have 


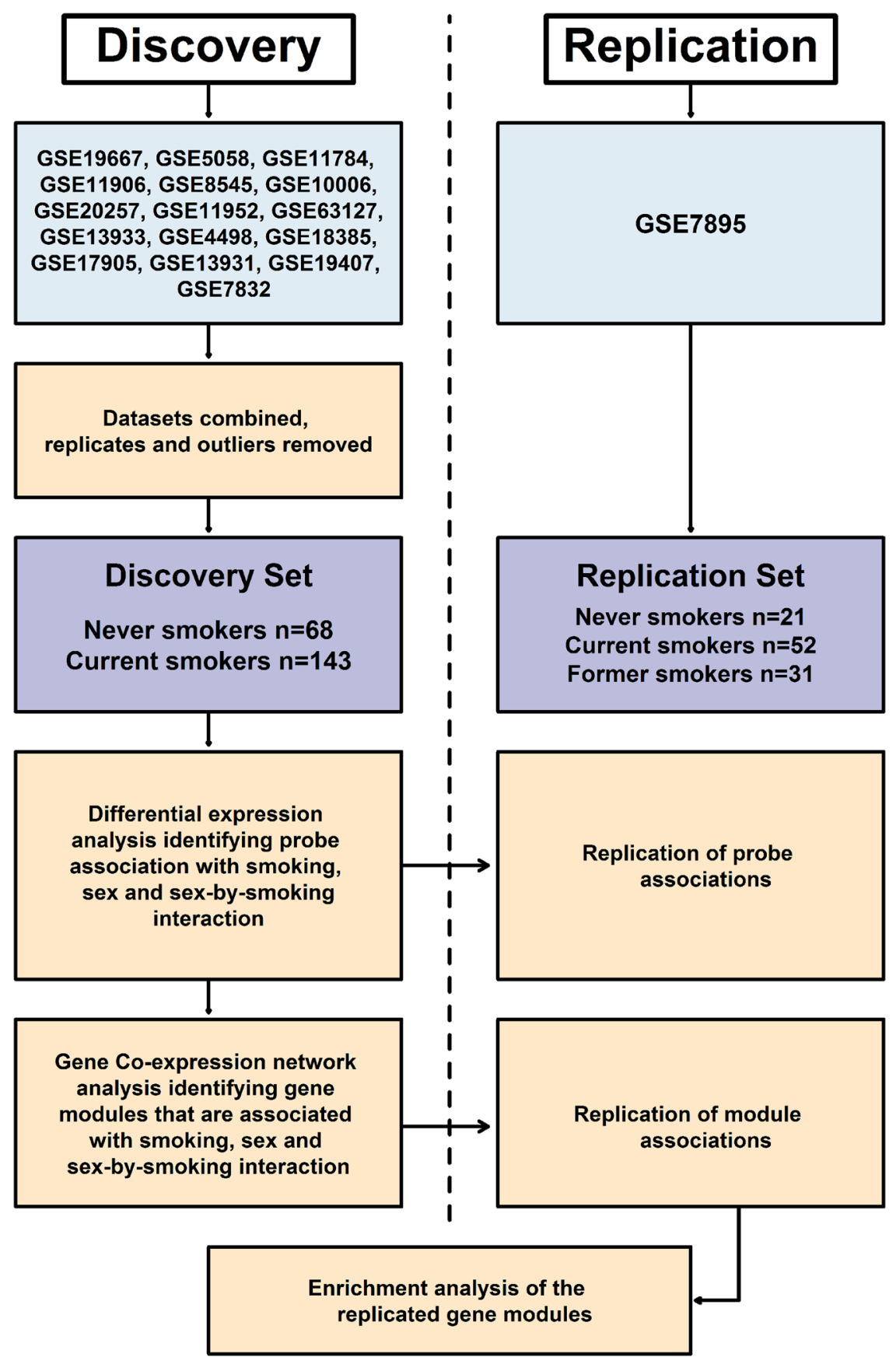

Figure 1. Overall study design.

enhanced ability to produce oxidants in the airways in response to cigarette smoking, leading to increased airway damage $e^{14,15}$.

To further understand the mechanisms underlying the sexual dimorphism in the pathogenesis of smoking related lung diseases, we examined the effects of sex, and its interaction with cigarette smoking, on the transcriptome of human airway epithelial cells.

\section{Results}

The overall study design is shown in Fig. 1. The demographics of the discovery and replication cohorts are described in Table 1 . The discovery set included 68 never smokers and 143 current smokers while the replication set included 21 never smokers, 31 former smokers and 52 current smokers. There were no statistically significant differences in age or pack-years between female never smokers, female current smokers, male never smokers and male current smokers in the discovery or the replication sets (all Bonferroni adjusted p-values $>0.05$ ). We also used a third dataset (GSE37147) for potential replication although it only contained data on former and current smokers (i.e. there were no never-smokers). However, compared to the discovery and replication sets, all except 


\begin{tabular}{|c|c|c|c|c|c|c|c|c|c|c|c|c|c|c|}
\hline \multirow{3}{*}{$\begin{array}{l}\text { Sex } \\
\begin{array}{l}\text { Smoking } \\
\text { Status }\end{array}\end{array}$} & \multicolumn{4}{|c|}{ Discovery Set } & \multicolumn{6}{|c|}{ Replication Set } & \multicolumn{4}{|l|}{ GSE37147 } \\
\hline & \multicolumn{2}{|c|}{$\begin{array}{l}\text { Female } \\
\mathrm{n}=70,33.2 \%\end{array}$} & \multicolumn{2}{|c|}{$\begin{array}{l}\text { Male } \\
n=141,66.8 \%\end{array}$} & \multicolumn{3}{|c|}{$\begin{array}{l}\text { Female } \\
\mathrm{n}=25,24.0 \%\end{array}$} & \multicolumn{3}{|c|}{$\begin{array}{l}\text { Male } \\
\mathrm{n}=79,76.0 \%\end{array}$} & \multicolumn{2}{|c|}{$\begin{array}{l}\text { Female } \\
n=103,43.3 \%\end{array}$} & \multicolumn{2}{|c|}{$\begin{array}{l}\text { Male } \\
n=135,56.7 \%\end{array}$} \\
\hline & \begin{tabular}{|l|}
$\mathrm{NS}$ \\
$\mathrm{n}=26$ \\
$37.1 \%$
\end{tabular} & \begin{tabular}{|l}
$\mathrm{CS}$ \\
$\mathrm{n}=44$, \\
$62.9 \%$
\end{tabular} & $\begin{array}{l}\mathrm{NS} \\
\mathrm{n}=42 \\
29.8 \%\end{array}$ & $\begin{array}{l}\mathrm{CS} \\
\mathrm{n}=99 \\
70.2 \%\end{array}$ & $\begin{array}{l}\text { NS } \\
n=5 \\
20.0 \%\end{array}$ & $\begin{array}{l}\mathrm{FS} \\
\mathrm{n}=10 \\
40.0 \%\end{array}$ & $\begin{array}{l}\mathrm{CS} \\
\mathrm{n}=10, \\
40.0 \%\end{array}$ & $\begin{array}{l}\text { NS } \\
n=16 \\
20.3 \%\end{array}$ & $\begin{array}{l}\mathrm{FS} \\
\mathrm{n}=21 \\
26.6 \%\end{array}$ & $\begin{array}{l}\mathrm{CS} \\
\mathrm{n}=42, \\
53.1 \%\end{array}$ & \begin{tabular}{|l|}
$\mathrm{FS}$ \\
$\mathrm{n}=54$ \\
$52.4 \%$
\end{tabular} & $\begin{array}{l}\mathrm{CS} \\
\mathrm{n}=49, \\
47.6 \%\end{array}$ & \begin{tabular}{|l|}
$\mathrm{FS}$ \\
$\mathrm{n}=85$ \\
$63.0 \%$
\end{tabular} & $\begin{array}{l}\mathrm{CS} \\
\mathrm{n}=50 \\
37.0 \%\end{array}$ \\
\hline Age & $(38.0,23.5)$ & $(46.0,12.5)$ & $(39.5,11.5)$ & $(45.0,7.5)$ & $(28.0,7.0)$ & $(44.0,11.8)$ & $(40.5,13.5)$ & $(29.5,14.5)$ & $(63.0,18.0)$ & $(47.5,25.3)$ & $(66.0,6.98)$ & $(63.7,6.5)$ & $(66.3,8.7)$ & $(61.1,7.37)$ \\
\hline Pack years & $(0.0,0.0)$ & $(28.5,16.8)$ & $(0.0,0.0)$ & $(26.0,21.8)$ & $(0.0,0.0)$ & $(6.0,25.8)$ & $(24.0,17.1)$ & $(0.0,0.0)$ & $(35.0,52.0)$ & $(25.0,39.4)$ & $(42.0,15.0)$ & $(47.1,15.1)$ & $(47.6,19.9)$ & $(43.2,13.8)$ \\
\hline COPD & $\mathrm{n}=0$ & $\mathrm{n}=11$ & $\mathrm{n}=0$ & $\mathrm{n}=31$ & NA & NA & NA & NA & NA & NA & $\mathrm{n}=21$ & $\mathrm{n}=14$ & $\mathrm{n}=36$ & $\mathrm{n}=16$ \\
\hline
\end{tabular}

Table 1. Demographics of subjects in the discovery and the replication set. Descriptive statistics are shown as (median, interquartile range). NS $=$ Never Smoker, FS $=$ Former Smoker, CS $=$ Current Smoker.

male former smokers in GSE37147 were significantly older (all Bonferroni adjusted p-values $<0.05$ ). The male current smokers in GSE37147 also had increased pack-years compared to those in the discovery or the replication sets (Bonferroni adjusted p-values $<0.05$ ). The female current smokers in GSE37147 had increased pack-years compared to those in the discovery set (Bonferroni adjusted $\mathrm{p}$-value $=9.04 \times 10^{-03}$ ) but not to those in the replication set (Bonferroni adjusted p-value $=0.95$ ).

Prediction of sex in the replication set. The best-performing elastic net-regularized logistic regression model consisted of three probes and together they demonstrated $100 \%$ cross-validated area under the receiver-operator characteristics curve (AUC) in the training dataset. These three probes corresponded to RPS4Y1, DDX3Y and KDM5D genes, which are located on the Y-chromosome. The model was then evaluated in the testing dataset and also showed perfect ability to discriminate with an AUC of 100\% (Supplementary Fig. 2A,B). After confirming the model performance, we applied it to the replication set to classify the subjects into 79 males and 25 females (Supplementary Fig. 2C).

Differential expression with sex and smoking. As shown in the principal component analysis (PCA) plot in Supplementary Fig. 1E, there was clear clustering in the gene expression profiles of the discovery set, the replication set, and GSE37147. The differences may be related to different microarray platforms that were used and different generations of the airway that were sampled across the three studies.

In the discovery set, 8,000 probes, corresponding to 5,466 genes, were differentially expressed between never and current smokers after adjustment for sex, age, COPD, ethnicity and pack-years of smoking $(\mathrm{FDR}<0.1)$ and 735 of these genes were replicated in the replication set (at a threshold of $\mathrm{P}<0.05$ ) (Fig. 2A,B). To identify and replicate genes, an FDR $<0.1$ was used in the hypothesis-free discovery analyses. For replication, a gene was considered "replicated" if any of the probes showed differential expression between never and current smokers in the replication cohort at $\mathrm{P}<0.05$ and with the same direction of effect. The top three replicated genes associated with smoking were $A D H 7\left(\mathrm{FDR}=1.15 \times 10^{-32}\right), G P X 2\left(\mathrm{FDR}=1.43 \times 10^{-31}\right)$ and $A L D H 3 A 1\left(\mathrm{FDR}=8.56 \times 10^{-31}\right)$, which were all up-regulated in current smokers. We also validated the discovery in a second replication set GSE37147; out of the 735 replicated genes, 526 were further replicated in GSE37147 study of former versus current smokers.

A total of 169 probes, corresponding to 100 genes, were differentially expressed between males and females after adjustment for smoking status, age, ethnicity and pack-years (FDR $<0.1)$, and 27 of these genes were replicated in the replication set $(\mathrm{P}<0.05)$ (Fig. 2C,D). Four of the replicated genes, CCND2, FABP5, NLRP2, and SERPINB3, are on the autosome (Fig. 3A). CCND2 and NLRP2 were down-regulated in males while FABP5 and SERPINB3 were up-regulated in males. The top three genes differentially expressed between males and females were RPS4Y1 $\left(\mathrm{FDR}=3.98 \times 10^{-88}\right), D D X 3 Y\left(\mathrm{FDR}=4.33 \times 10^{-86}\right)$ and $E I F 1 A Y\left(\mathrm{FDR}=6.79 \times 10^{-82}\right)$, which are located on the Y-chromosome (all three genes were up-regulated in males). Additional evaluation using GSE37147 replicated 22 out of the 27 replicated genes, including CCND2 and NLRP2.

The complete lists of genes that were significantly associated with smoking status and sex are shown in Supplementary Tables 2 and 3, respectively.

Sexually dimorphic gene expression changes associated with smoking. Having identified genes differentially expressed according to sex and smoking status, we next identified sexually dimorphic genes in response to smoking. In the discovery set, 3,416 probes corresponding to 2,654 genes were associated with sex-by-smoking interaction (FDR $<0.1$ ) (Fig. 2E). In the replication set, 1,737 probes corresponding to 1,423 genes were associated with sex-by-smoking interaction $(\mathrm{P}<0.05)$ (Fig. $2 \mathrm{~F})$. A total of 184 genes showed differential expression between never and current smokers in both datasets and within the same direction (Fig. 4A). The top three replicated genes were RALGDS $\left(\mathrm{FDR}=2.19 \times 10^{-03}\right), D N A I 2\left(\mathrm{FDR}=9.84 \times 10^{-03}\right)$ and $D G K Z$ $\left(\mathrm{FDR}=1.48 \times 10^{-03}\right)$. The sexually dimorphic genes in response to smoking were not restricted to the XY chromosome; $96.5 \%$ of the associated genes in the discovery set and $94.0 \%$ of the 184 replicated genes were located on the autosome (Fig. 3B). The lists of probes that were associated with the sex-by-smoking interaction term are shown in Supplementary Table 4 in the discovery and the replication sets. Interestingly, the number of replicated genes decreased to 26 when we combined the never and former smokers together and compared them with the current smokers in the replication set, despite the increase in sample size (Fig. 4B). The number of replicated genes decreased to 9 if we compared the former smokers to the current smokers in the replication set (Fig. 4C). Similarly, when GSE37147 (containing current and former smokers but no never-smokers) was used as the replication set, only 14 genes were replicated (Fig. 4D). Figure 4E shows the expression of the top three replicated 
A

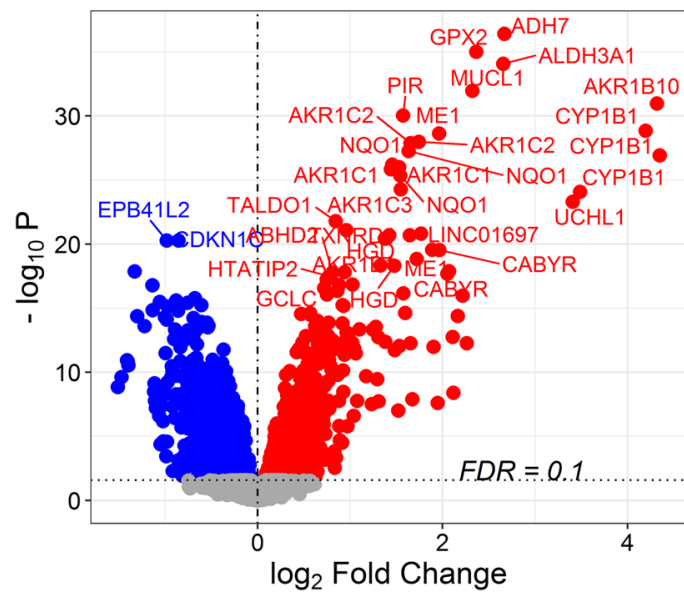

C

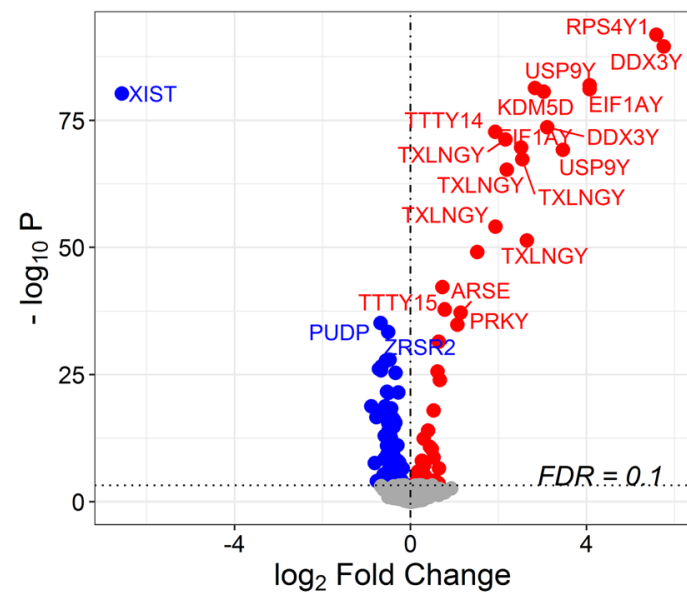

E

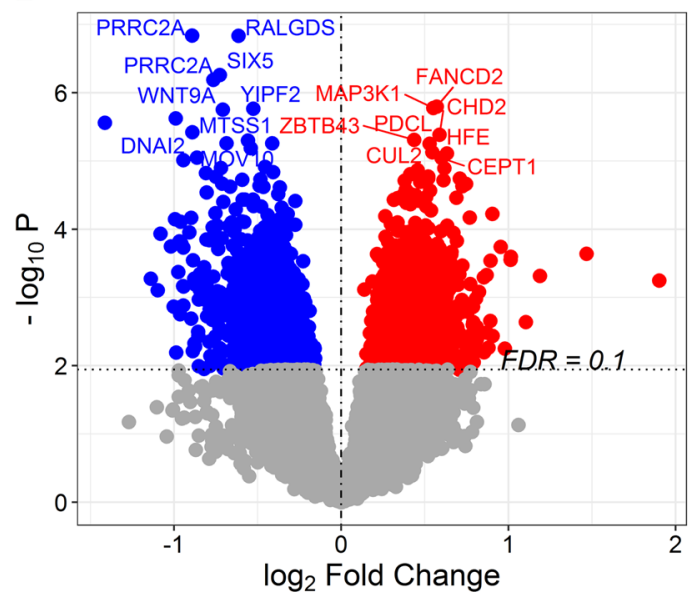

B

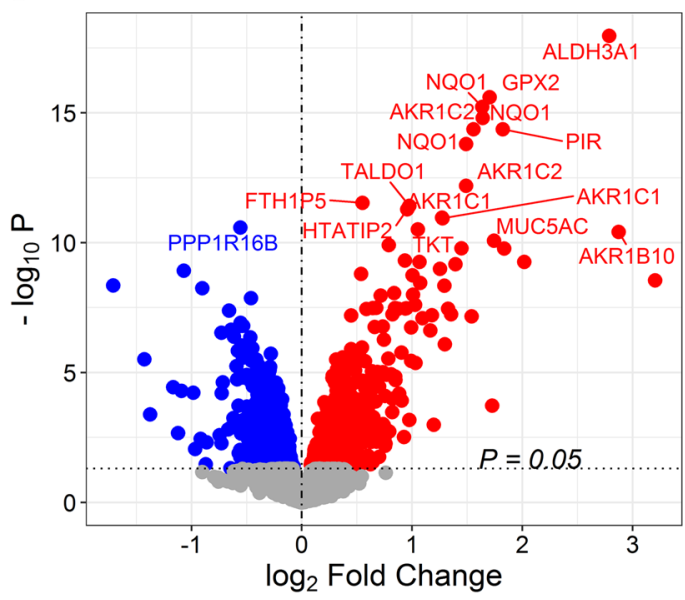

D

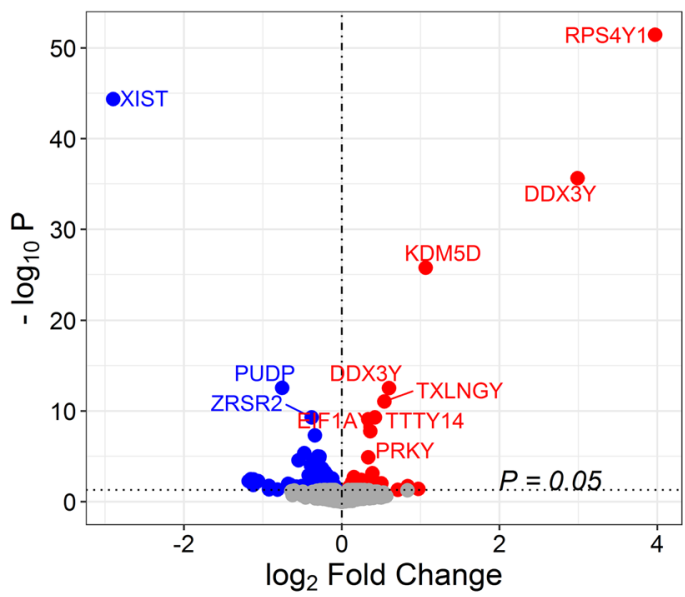

$\mathbf{F}$

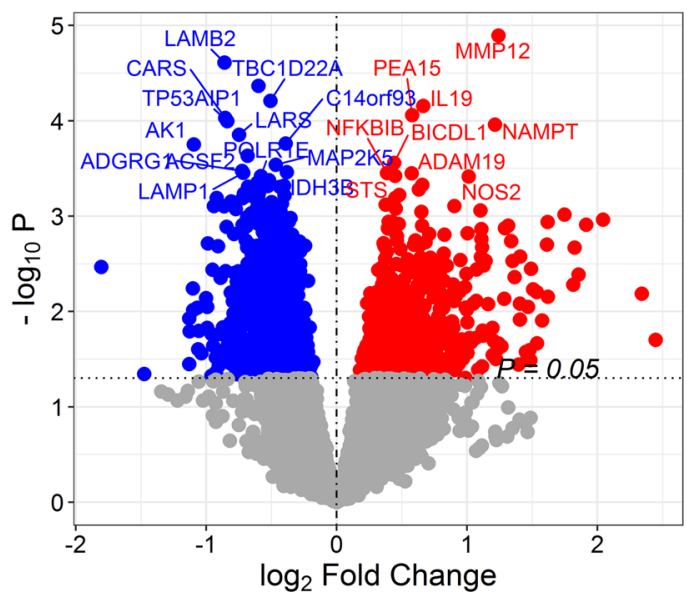

Figure 2. Volcano plots showing probe-phenotype associations. (A) Probe associations for smoking status in the discovery set. (B) Probe associations for smoking status in the replication set. (C) Probe associations for sex in the discovery set. (D) Probe associations for sex in the replication set. (E) Probe associations for sex-by-smoking interaction in the replication set. (F) Probe associations for sex-by-smoking interaction in the discovery set. Probes shown in red were up-regulated in current smokers and probes shown in blue were down-regulated in current smokers in $(\mathbf{A})$ and $(\mathbf{B})$. Probes shown in red were up-regulated in males and probes shown in blue were down-regulated in males in (C) and (D). Probes shown in red exhibit a positive sex-bysmoking interaction in male current smokers while the probes shown in blue exhibit a negative sex-by-smoking interaction in male current smokers in $(\mathbf{E})$ and $(\mathbf{F})$. 

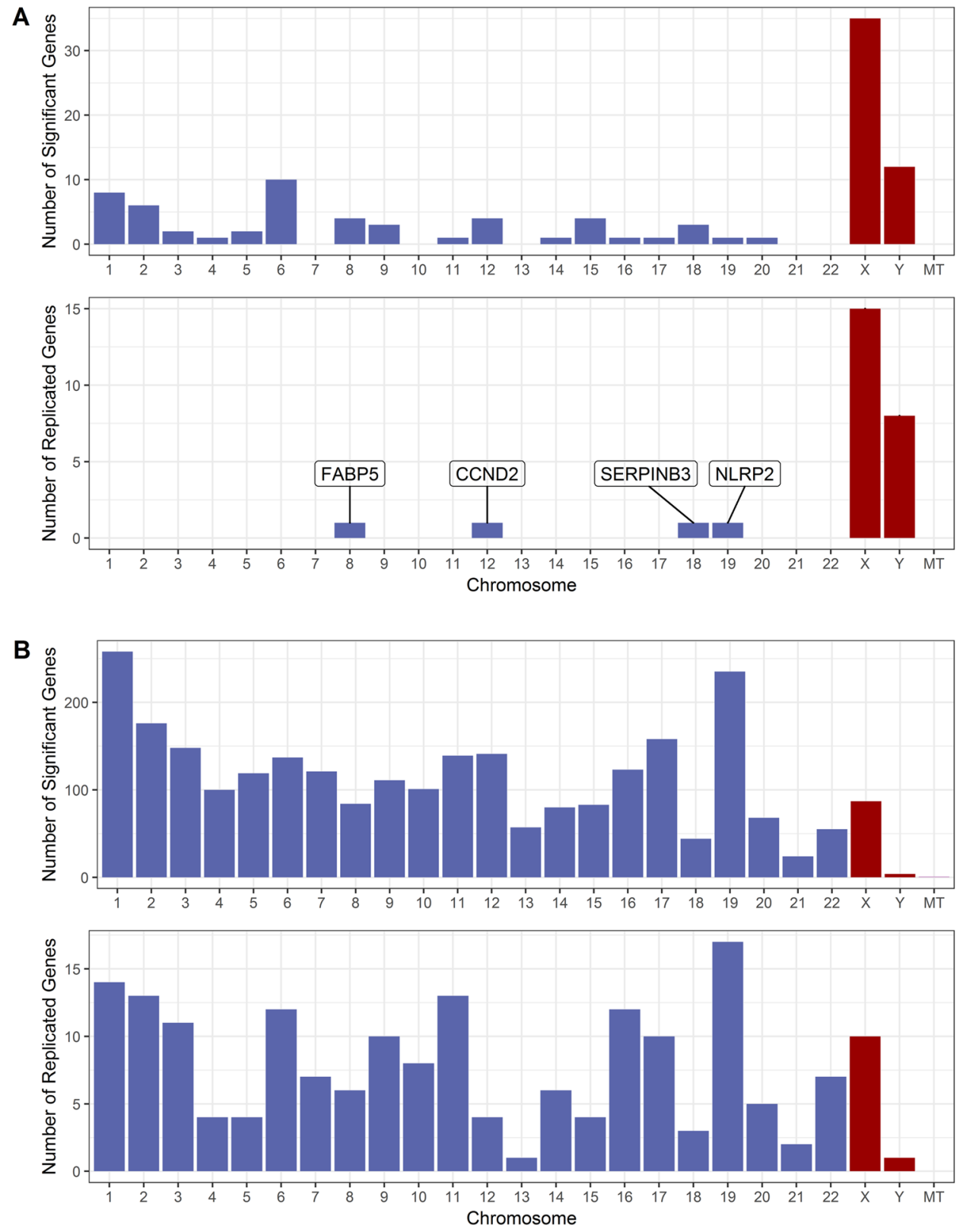

Figure 3. Chromosome distribution of genes that were associated with sex or sex-by-smoking interaction. (A) Top: Chromosome distribution of genes associated with sex in the discovery set (FDR $<0.1)$. Bottom: Chromosome distribution of the replicated genes for sex. (B) Top: Chromosome distribution of genes associated with sex-by-smoking interaction in the discovery set (FDR $<0.1)$. Bottom: Chromosome distribution of the replicated genes for sex-by-smoking interaction. Abbreviation used in the figure: $\mathrm{MT}=$ mitochondrion.

genes in both the discovery and the replication set (never vs. current smokers). If we use GSE37147 to further replicate the 184 replicated genes, only one gene, PLCB2, was also replicated in GSE37147.

Module-level associations. Based on the expression of 12,537 genes in the discovery set, 37 gene modules were constructed. The module sizes ranged from 17 genes in module 36 to 3,822 genes in module 01 . Eleven modules were significantly associated with smoking status (never vs. current smokers) after adjusting for age, sex, ethnicity and pack-years of smoking $($ FDR $<0.1)$ in the discovery set. Four of these modules - module 22, module 11, module 17, and module 15 - were successfully replicated in the replication set (never and former vs. current smokers) at a threshold of $\mathrm{P}<0.05$.

Only module 36 was associated with sex $(F D R<0.1)$ in the discovery set and it was also replicated in the replication set at a threshold of $\mathrm{P}<0.05$.

Thirteen modules were associated with sex-by-smoking interaction in the discovery set (FDR $<0.1)$. Three modules - module 02 , module 34 and module 36 - were successfully replicated in the replication set $(\mathrm{P}<0.05)$. Module 34 and module 36 were relatively small in size with 18 and 17 genes, while module 02 included 1,860 
A

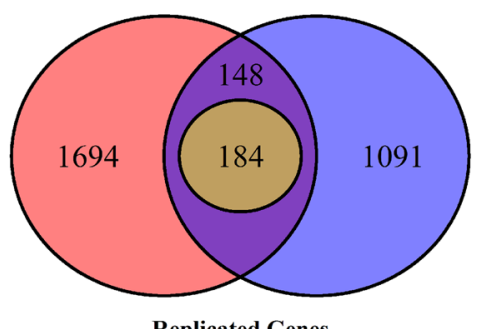

C Discovery Set (NS vs. CS) Replication Set (FS vs. CS)

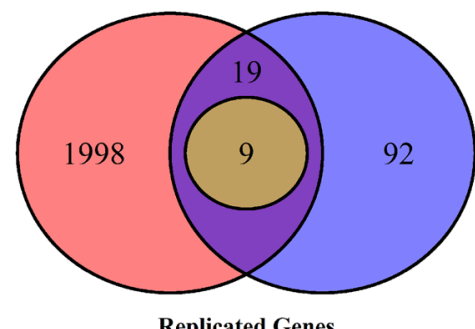

E

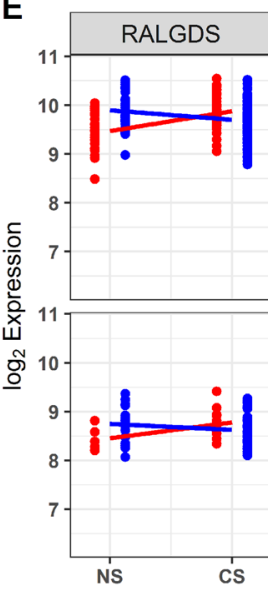

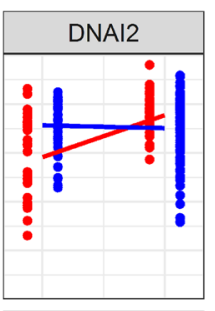
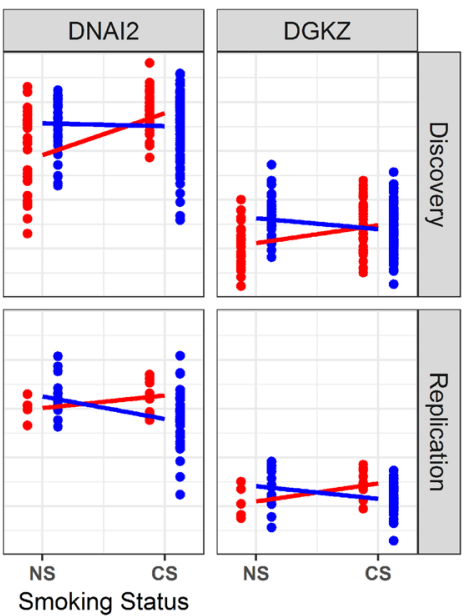

Sex $\rightarrow$ Female $\rightarrow$ Male

Figure 4. Replication of genes that were associated with sex-by-smoking interaction. (A) Venn diagram showing the number of associated genes in the discovery set (NS vs. CS) and in the replication set (NS vs. CS). (B) Venn diagram showing the number of associated genes in the discovery set (NS vs. CS) and in the replication set (NS and FS combined together vs. CS). (C) Venn diagram showing the number of associated genes in the discovery set (NS vs. CS) and in the replication set (FS vs. CS). (D) Venn diagram showing the number of associated genes in the discovery set (NS vs. CS) and in GSE37147 (FS vs. CS). (E) Gene expression of the top 3 replicated genes for sex-by-smoking interaction in the discovery and the replication set. Abbreviations used in the figure: NS = Never Smoker, FS = Former Smoker, CS = Current Smoker.

genes. The module-phenotype association along with the module sizes for the replicated modules are shown in Fig. 5. The associations between the modules and the phenotypes are shown in Supplementary Table 5.

Gene set variation analysis (GSVA). In addition to the gene-level and the WGCNA network-level differential expression analyses, we also performed GSVA to replicate the identified gene signatures in the replication cohort. As mentioned previously we have identified 2,654 genes and 13 gene modules associated with sex-by-smoking interaction using gene- and network-level analysis, respectively. The 2,654 genes were considered as one gene set, and each of the 13 modules were considered as a separate gene set. Using GSVA, we found that 


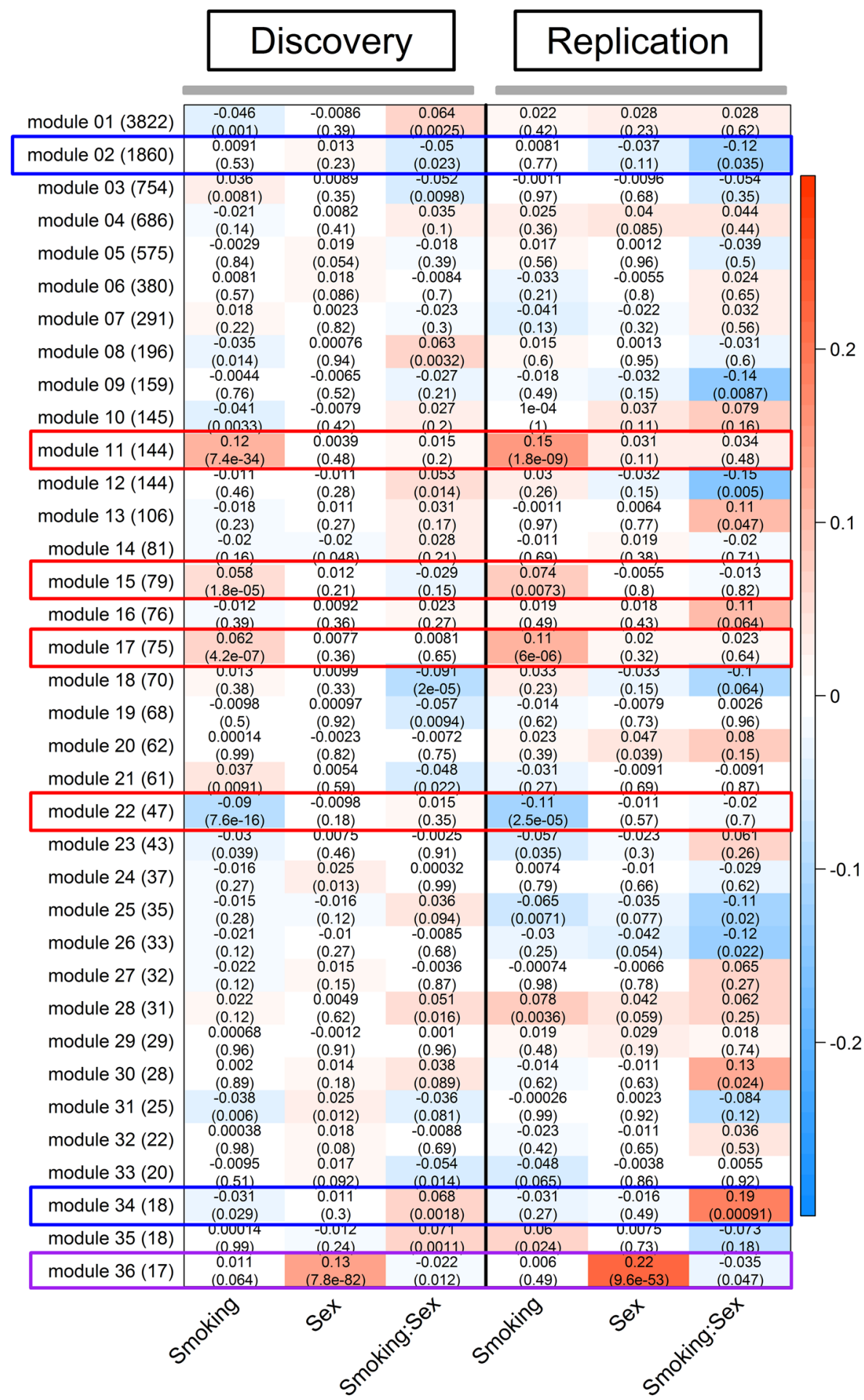

Figure 5. Module-level associations in the discovery and replication sets. The rows represent the gene modules and the sizes of the modules are shown within parentheses next to the module names. The columns represent the phenotypes. In each cell, the number at the top is the linear regression coefficient and the number in parentheses is the corresponding $\mathrm{p}$-value. The color of the cell is proportional to the regression coefficient. From column 1 to column 3 of both the discovery and the replication sets, the red color indicates (1) up-regulation in current smokers, (2) up-regulation in male subjects, and (3) a positive sex-by-smoking interaction effect in male current smokers. The replicated modules have been highlighted (red for smoking effect, blue for smoking-bysex interaction and purple for both sex effect and smoking-by-sex interaction). 


\begin{tabular}{|c|c|c|}
\hline ID & GO biological process & FDR \\
\hline \multicolumn{3}{|c|}{ module 22 (smoking) } \\
\hline GO:0031333 & negative regulation of protein complex assembly & $1.77 \times 10^{-02}$ \\
\hline \multicolumn{3}{|c|}{ module 11 (smoking) } \\
\hline GO:1901661 & quinone metabolic process & $6.30 \times 10^{-06}$ \\
\hline GO:0006081 & cellular aldehyde metabolic process & $6.30 \times 10^{-06}$ \\
\hline GO:0019748 & secondary metabolic process & $1.86 \times 10^{-05}$ \\
\hline GO:0098869 & cellular oxidant detoxification & $2.05 \times 10^{-05}$ \\
\hline GO:0034754 & cellular hormone metabolic process & $4.09 \times 10^{-05}$ \\
\hline \multicolumn{3}{|c|}{ module 17 (smoking) } \\
\hline \multicolumn{3}{|c|}{ No significant enrichment. } \\
\hline \multicolumn{3}{|c|}{ module 15 (smoking) } \\
\hline GO:0070268 & cornification & $4.60 \times 10^{-02}$ \\
\hline GO:0006486 & protein glycosylation & $4.60 \times 10^{-02}$ \\
\hline GO:0043413 & macromolecule glycosylation & $4.60 \times 10^{-02}$ \\
\hline GO:0016266 & O-glycan processing & $4.60 \times 10^{-02}$ \\
\hline GO:0046718 & viral entry into host cell & $4.60 \times 10^{-02}$ \\
\hline \multicolumn{3}{|c|}{ module 36 (sex and smoking:sex) } \\
\hline GO:0006413 & translational initiation & $1.12 \times 10^{-03}$ \\
\hline GO:0006687 & glycosphingolipid metabolic process & $4.80 \times 10^{-02}$ \\
\hline GO:0006614 & SRP-dependent cotranslational protein targeting to membrane & $4.80 \times 10^{-02}$ \\
\hline GO:0006613 & cotranslational protein targeting to membrane & $4.80 \times 10^{-02}$ \\
\hline GO:0045047 & protein targeting to ER & $4.80 \times 10^{-02}$ \\
\hline \multicolumn{3}{|c|}{ module 02 (smoking:sex) } \\
\hline GO:0006914 & autophagy & $2.91 \times 10^{-06}$ \\
\hline GO:0061919 & process utilizing autophagic mechanism & $2.91 \times 10^{-06}$ \\
\hline GO:0000422 & autophagy of mitochondrion & $9.42 \times 10^{-05}$ \\
\hline GO:0061726 & mitochondrion disassembly & $9.42 \times 10^{-05}$ \\
\hline GO:0035966 & response to topologically incorrect protein & $4.82 \times 10^{-04}$ \\
\hline \multicolumn{3}{|c|}{ module 34 (smoking:sex) } \\
\hline GO:0009615 & response to virus & $1.29 \times 10^{-14}$ \\
\hline GO:0045071 & negative regulation of viral genome replication & $2.53 \times 10^{-07}$ \\
\hline GO:0043900 & regulation of multi-organism process & $3.25 \times 10^{-07}$ \\
\hline GO:0019079 & viral genome replication & $6.30 \times 10^{-06}$ \\
\hline GO:0060337 & type I interferon signaling pathway & $2.52 \times 10^{-03}$ \\
\hline
\end{tabular}

Table 2. Top GO biological processes enriched in each replicated module. The associated effect is shown in brackets.

the gene set with the 2654 genes was not significantly related to sex-by-smoking interaction in the replication cohort $(\mathrm{P}=0.660)$ while module 34 and module 28 were significantly associated with sex-by-smoking interaction ( $\mathrm{P}=0.016$ and $\mathrm{P}=0.039$, respectively) in the replication cohort.

Enrichment in biological processes. An FDR cutoff of 0.1 was used to identify Gene Ontology (GO) biological processes that were associated with the replicated modules. Among modules that were associated with smoking status, module 22 genes were associated with protein complex assembly and the top genes with the highest module membership (MM) in this module were NPAS3, CYP4X1 and PGRMC1. Module 11 genes were associated with metabolic processes, responses to oxidative stress and homeostasis and the top members of this module were $A K R 1 B 10, G P X 2, A K R 1 C 2$. Module 15 genes were related to cornification, protein glycosylation and viral entry into host cell. The top members of the module 15 module are ST14, HM13, TMPRSS4. No significant pathways were enriched in module 17 and the top members of this module were CEACAM5, GALE and AGR2.

Of the three modules associated with sex-by-smoking interaction, all the genes in module 36 belonged to the $\mathrm{X}$ and $\mathrm{Y}$ chromosomes and showed enrichment in translational initiation, protein targeting to endoplasmic reticulum, and histone demethylation. The top members of module 36 were DDX3Y, KDM5D and USP9Y. This module was also associated with sex. For the remaining two modules, module 34 showed strong association with defense responses to virus, regulation of innate immune response, membrane fusion, and Th2 cell cytokine production. The genes with the highest MM in module 34 were DDX60, SAMD9 and IFI44. Module 02 was related to autophagy, biological processes of mitochondrion, cellular response to oxidative stress, and negative regulation of the MAPK signaling cascade. The top members of module 02 were RALY, UQCRC1 and MAP2K2. Table 2 shows the top GO biological processes enriched in the replicated modules and Supplementary Table 6 shows the complete enrichment results for the replicated modules. Supplementary Table 7 shows the list of top 10 genes with the highest membership in each module. 
A

A Autophas

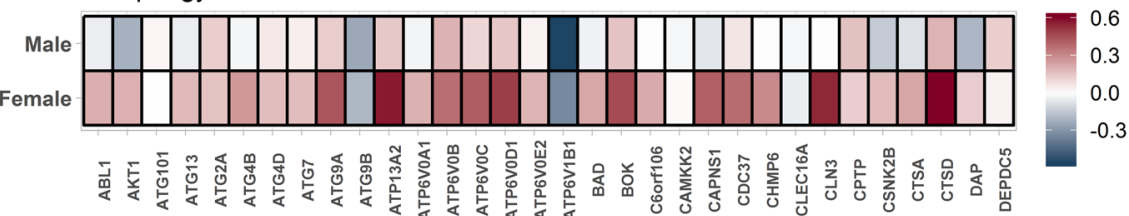

$\log _{2}$ Fold Change

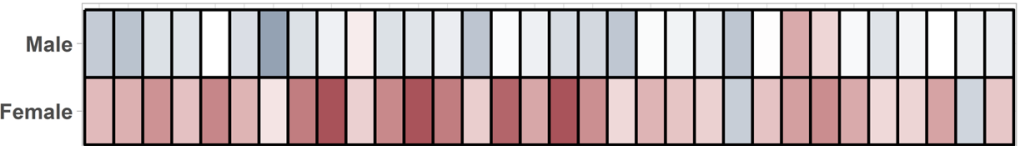

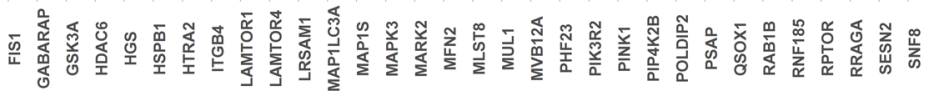

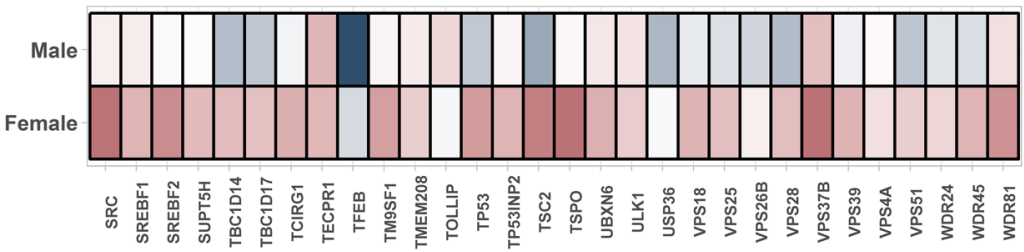

B

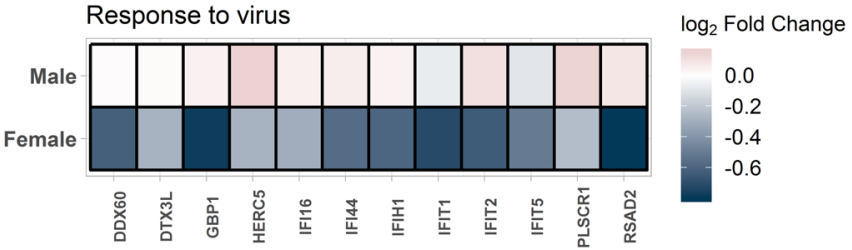

Figure 6. Sexually dimorphic effect of smoking on gene expression of genes in the top GO biological process in (A) module 02 and (B) in module 34. The red color indicates up-regulation in current smokers and the blue color indicates down-regulation in current smokers.

In addition to the modules that were replicated through WGCNA, module 28, which was associated with sex-by-smoking interaction and replicated using GSVA, was associated with the amino sugar biosynthetic process $(\mathrm{FDR}=0.080)$.

In Fig. 6, the gene expression changes for genes in the significant pathways (autophagy and response to virus) are shown in males and females separately in response to smoking. While genes in autophagy pathway show little change in males, they were significantly up-regulated in females in response to smoking. On the other hand, genes in the viral response pathway showed little change in males but were significantly down-regulated in females in response to smoking.

Enrichment in transcription factor binding site (TFBS). A FDR cutoff of 0.05 and an odds ratio $>2$ were used to identify TFBSs that were enriched in genes in the replicated modules. For modules that were associated with smoking status, 13 TFBSs were enriched in module 22, 8 TFBSs were enriched in module 11, no TFBSs were enriched in module 17, and 1 TFBS was enriched in module 15 . No TFBS was enriched in module 36, which was associated with both sex and sex-by-smoking interaction. For the remaining two modules that were associated with sex-by-smoking interaction, 72 TFBSs were enriched in module 02 including estrogen-related receptor alpha (ESRRA), and 7 TFBSs were enriched in module 34. Supplementary Table 8 shows the complete list of enriched TFBSs in each module.

Comparing the identified sex-by-smoking gene signature with previous literature. Recently, van den Berge et al. identified a sex-by-COPD gene expression signature in human whole lung tissue ${ }^{16}$. Since the full gene expression signature is not available, we were only able to compare the top 5 most significant genes for positive sex-by-COPD interaction (SGMS1, LINGO2, SLC25A1, KDSR, SKP1) and the top 5 most significant genes for negative sex-by-COPD interaction (CYorf15A, ZFY, AMPD3, STAP1, MMP13) against our sex-by-smoking interaction results in airway epithelium.

We first checked these 10 genes in our discovery cohort. At FDR $<0.1, S G M S 1$ and SKP1, which were down-regulated with COPD in females, were also down-regulated with smoking in females; KDSR, which showed both down-regulation with COPD in females and up-regulation with COPD in males, was down-regulated with smoking in females while remaining unchanged with smoking in males. However, none of these genes were replicated in the replication cohort at $\mathrm{P}<0.05$. Perhaps it is not surprising to see differences between our gene signatures and the gene signature reported by van den Berge et al. because the tissues are different (airway 
epithelium vs. whole lung tissue) and COPD genes are not necessarily the same as smoking genes as we have shown previously ${ }^{17}$.

We also checked our gene signature against the study reported by Engle et al. ${ }^{18}$, in which both male and female mice were exposed to smoke or sham (room air). We downloaded the data from the GEO (under accession GSE109776) and performed a differential expression analysis on sex-by-smoke exposure interaction similarly while adjusting for the length of smoke exposure. Of the 184 replicated genes that we identified to be associated with sex-by-smoking interaction in human airway epithelium in our study, only 7 genes were associated with sex-by-smoke exposure in mouse lung tissue in the study of Engle et al. at the $\mathrm{P}<0.05$ threshold.

\section{Discussion}

Studying the transcriptome is a very powerful hypothesis-free approach to elucidate the molecular mechanisms underlying diseases and phenotypes. In this study, we analyzed the differential transcriptomics of human airway epithelium associated with smoking as well as the transcriptomics of smoking differentially between males and females. We found that 1) smoking has a large and reproducible effect on gene expression profiles; 2) the smoking effects on the transcriptome of human airways are modified by the underlying sex of the individual; 3 ) the sexually dimorphic smoking genes are common throughout the autosome and not restricted to sex chromosomes; and 4) the sexually dimorphic smoking genes are enriched in biological processes that are related to anti-viral responses, type 1 interferon signaling, and autophagy.

There is increasing interest in understanding sexual dimorphisms across organs and tissues. In the study of Melé et al. ${ }^{19}$, the authors investigated sexual dimorphism in gene expression data of 1,641 samples from 175 individuals representing 43 sites and identified 753 genes that had tissue specific sex-biased expression $($ FDR $<0.05)$. Chen et al..$^{20}$ used a much larger sample size (compared to Melé et al.) and investigated sexual dimorphism in gene expression data from the GTEx dataset: they evaluated 8,716 human transcriptomes collected from 31 tissues in 549 individuals of whom 188 (34\%) were female. The authors observed sexually dimorphic gene expression for $10 \%$ of the genes across autosomes in most tissues including the lung. The most sexually dimorphic gene expression was noted in the breast, skin, thyroid, brain, and adipose tissues, while the least dimorphic was in the gastrointestinal tract ${ }^{20}$. Multiple other studies in single cell or tissue types have confirmed to varying extents the large transcription differences between the two sexes ${ }^{21-24}$. Similar patterns emerged from these studies including the presence of large and perhaps underestimated differences in the transcriptome between males and females present throughout the autosomes and not restricted to sex chromosomes. Extrapolating findings from the published studies (even the large ones) to respiratory diseases is challenging since they do not contain data on airway epithelium and lack essential phenotypic information such as smoking. To our knowledge, the current study is the first to report sexually dimorphic gene expression changes in human airway epithelium associated with cigarette smoking. The airway epithelium is the initial site of injury in airway diseases such as COPD and is an important mediator of the host's immune response to inhaled toxins, allergens and medications ${ }^{25}$.

We found that smoking differentially altered two main pathways in men versus women. These included those related to the host response to viruses (e.g. type 1 interferon signaling) and autophagy. In females, our study revealed that most autophagy genes were up-regulated in response to smoking compared to males where these genes were either down-regulated or only slightly up-regulated (Fig. 6A). The highly-conserved process of autophagy involves the formation of intracellular vacuoles derived from the cell membrane (autophagosomes), which engulf and transport a diverse range of intracellular materials including protein aggregates, organelles, and intracellular bacteria ${ }^{26}$. Although autophagy is considered a normal homeostatic mechanism, its dysregulation has been linked to a number of diseases ${ }^{27}$. In COPD, lung tissue samples show increased vacuolization consistent with upregulated autophagy ${ }^{28}$. However, the role of autophagy in COPD pathogenesis, and its interaction with cigarette smoke (CS) exposure, is complex and may be cell-specific ${ }^{29}$. For example, CS has been shown to induce autophagy-related genes and proteins ${ }^{28,30}$, and increased autophagic flux may contribute to CS-induced epithelial cell death ${ }^{31}$. In contrast, deficiencies in CS-induced autophagy may contribute to CS-induced epithelial cell senescence in $\mathrm{COPD}^{32}$ and alveolar macrophages of smokers showed impaired autophagy (which may provide a mechanistic link to increased pulmonary infections in this population) ${ }^{33}$. Nevertheless, we have shown that autophagy-related genes are overall upregulated in women compared to men, in association with the sex-smoking interaction but not sex alone. While sexual dimorphism in autophagy is well-documented ${ }^{34,35}$, our results suggest that sex differences in autophagic processes may be the results of differential responses to CS exposure, rather than sex differences in constitutive autophagy. The functional consequences of our findings for COPD pathogenesis are difficult to predict since (1) the autophagy pathway is complex, and its genetic regulation is poorly understood ${ }^{36}$, (2) it is highly likely that both positive and negative autophagy regulators are differentially expressed ${ }^{37}$, and (3) whether differences in the transcriptome lead to differences in autophagy-related proteins cannot be determined from our study. Our findings should therefore serve as a catalyst for future studies into sex differences in autophagy.

The other sexually dimorphic pathway in response in smoking was type 1 interferon signaling and response to virus. Three of the downregulated genes in females while being unchanged or slightly upregulated in male smokers in Fig. 6B (IFIT1, IFIT2 and RSAD) are part of the type 1 interferon signaling pathway and are known to have antiviral activity ${ }^{38,39}$. The IFIT1 and IFIT2 genes hinder the expression of viral messenger RNAs by inhibiting the formation of the ribosomal preinitiation complex ${ }^{40}$, while the RSAD2 gene inhibit multiple DNA and RNA viruses including the human cytomegalovirus ${ }^{39}$ and the human immune deficiency virus (HIV $)^{41}$, hence it is possible that downregulation of IFIT1, IFIT2 and RSAD could negatively affect the immune response to viruses in female smokers when compared to male smokers. Previous studies have demonstrated that smoking affects the lung epithelium's ability to produce the early inductive and amplification phases of the type I interferon response and decreases the ability to elicit an antiviral response ${ }^{42,43}$. Furthermore, these effects were reversible and are almost completely abrogated with the antioxidant glutathione ${ }^{42}$. Sexual dimorphism in the immune response to viruses is well described (reviewed $\mathrm{in}^{44,45}$ ). The reasons underlying this sexually dimorphic response is attributed 
to sex hormones, sex chromosome-linked genes involved in viral responses, or genes on autosomes that seem to be regulated by sex chromosome-linked genes ${ }^{44}$. Certain sexually dimorphic immune responses are, however, present throughout life, and not necessarily between the years of puberty and reproductive senescence, suggesting that both genes and hormones are involved ${ }^{45}$. In general, females are thought to mount a stronger immune response to viral infections compared to males due to a more robust humoral and cellular immune response resulting in higher male mortality observed in epidemiological studies ${ }^{46}$. This is also consistent with observations that females have a higher risk to many autoimmune diseases ${ }^{47}$.

We performed enrichment analyses in transcription factor binding sites to determine whether or not the observed transcriptional differences are due to response to hormones and as such enriched for hormone receptor binding sites. We observed an enrichment in many transcription factors for the autophagy module including the estrogen-related receptor alpha ESRRA (enrichment FDR $=7.02 \times 10^{-33}$ ) suggesting the biological differences are not only due to the hormonal effect. Further studies are needed to understand the clinical consequences of this sexually dimorphic virus response to smoking with regard to COPD risk and progression, as well as exacerbations attributed to viral and bacterial infections. The finding of down-regulation of anti-viral response pathway genes in females in response to smoking is consistent with the epidemiological observations of higher exacerbation rate in females compared to males ${ }^{7}$.

We have used both gene- and network-level analyses in this study. The network-level approach to transcriptomic data is biologically intuitive since it takes into account the phenomenon of gene co-expression. We have previously used a network approach using WGCNA to identify a reproducible signature for lung function in peripheral blood ${ }^{48}$. The approach allows the identification of "hub" genes that reflect the biological processes of their corresponding modules. For example, the "hub" gene for the virus response enriched module (module 34) is DDX60 (DExD/H-box helicase 60), a gene that is involved in antiviral response ${ }^{49}$.

The definition of smoking has a big effect on discovery and replication of sexually dimorphic gene expression in response to smoking. When we included the data from GSE37147 (Sterling et al.) which included former and current smokers (but no never smokers) for replication, although the sample size is large $(n=238)$, the replication was poor and hence was not used for replication (Fig. 4D). Similarly, when we included both former and never smokers in our replication cohort vs. current smokers, and despite the increase in sample size, fewer genes were actually replicated compared to when we only included never vs. current smokers (Fig. 4A-C). This is consistent with previous findings from our group highlighting the importance of case definition of smokers on gene signature discovery ${ }^{50}$.

This study has a number of limitations. First, the sample size, although large, may not be sufficient to detect and replicate genes with smaller effects. Second, we used self-reported smoking status based on available data on GEO but self-reports may not accurately reflect smoking habits ${ }^{50}$. Third, we studied changes in airway epithelium given its role in the pathogenesis of lung diseases, but smoking may also affect other cell types such as immune and infiltrating cells in the airways. Finally, changes in gene expression may not necessarily reflect changes in protein levels or activities.

In summary, our study demonstrated that while smoking has a large effect on the transcriptome of airway epithelium, it differentially affects the viral response and autophagy pathways in female smokers compared to males. Such differential expression may explain the increased risk of disease and exacerbation among female smokers. These findings will stimulate mechanistic studies on how sex-specific genes impact the risk of smoking related diseases such as COPD.

\section{Methods}

Data collection. The search terms "airway epithelium" was used to identify studies of human airway epithelium gene expression from the Gene Expression Omnibus (GEO). After excluding irrelevant studies such as cancer, virus infection, influenza and injury, we identified 18 studies with phenotypic information on subjects' sex, smoking status and pack-years of smoking. Information on these 18 studies is shown in Supplementary Table 1. Of the studies identified, 16 were reported by the same lab and generated using the same microarray platform (U133A Plus 2.0 array). These datasets were combined together to form the "discovery" set. Phenotypic information such as sex, smoking status, age, COPD status, ethnicity and pack-years were available for 211 subjects (never smokers $n=68$; current smokers $n=143$ ) after removing duplicate data and outliers. GSE7895, which was generated using the U133A array, was used as the "replication" set consisting of 21 never smokers, 52 current smokers, and 31 former smokers. Smoking status, age and pack years were available from GEO for GSE7895 but the sex information was missing which was imputed for this cohort based on gene expression data from the discovery cohort (see below). GSE37147, which was generated using the GeneChip Human Gene 1.0 ST Array, was used as an additional replication cohort. Smoking status, sex, pack-years and lung function measurements of forced expiratory volume in 1 second $\left(\mathrm{FEV}_{1}\right)$ and $\mathrm{FEV}_{1} /$ forced vital capacity $\left(\mathrm{FEV}_{1} / \mathrm{FVC}\right)$ were available for 99 current smokers and 139 former smokers. Figure 1 shows a flowchart of the overall study design.

Data processing. Raw data (.CEL files) were downloaded from GEO for the discovery set, the replication set and GSE37147. Since the 16 studies of the discovery set contained overlapping samples, we carefully examined the phenotypic information of each sample to remove biological and technical replicates. No batch effect was detected among these studies using PCA (Supplementary Fig. 1A). The raw data of the discovery set were then normalized together using the Robust Multi-array Average (RMA) method. Similar to the discovery set, the replication set was also normalized using the RMA method (Supplementary Fig. 1B). The presence of outliers was assessed using a PCA before and after normalization for both the discovery and replication sets. Three outliers were observed and removed from the discovery set (Supplementary Fig. 1C) while no obvious outliers were observed in the replication set (Supplementary Fig. 1D). GSE37147 was also processed similarly and no obvious batch effect or outliers were detected. Large differences in the gene expression profiles were observed among the discovery set, the replication set and GSE37147 (Supplementary Fig. 1E). All data processing and statistical analysis were performed within the R statistical computing environment (version 3.5.0). 
Prediction of sex in the replication set. Since the sex information of the replication set was not available from GEO, we imputed the sex of subjects using gene expression of selected XY-chromosome probes. To identify the probes that best discriminated male from female subjects, elastic net-regularized logistic regression was used for probe selection. The discovery set was partitioned into a training dataset, which contained $70 \%$ of female subjects and an equal number of male subjects, and a test dataset, which contained the remaining participants. Models with parameters were built based on the training dataset. The AUC obtained from 100 repeats of a 10 -fold cross-validation was used as the criterion to evaluate the performance of the models. The performance of the model with the highest AUC was validated in the testing dataset. Finally, the model was applied to the replication set to predict the sex information.

Comparing the demographic characteristics. We performed Kruskal-Wallis H test followed by Dunn's test with Bonferroni correction to compare age and pack-years of smoking between males and females within different smoking status strata in the discovery set, the replication set, and GSE37147.

Modeling the effect of smoking and sex on gene expression. Linear regression was used to identify probes that were associated with smoking status, sex and sex-by-smoking interaction adjusting for potential covariates such as age, ethnicity and pack-years of smoking in both the discovery and replication sets. The analyses were performed using $\mathrm{R}$ package limma ${ }^{51}$. The Benjamini-Hochberg method was used to control the false discovery rate (FDR) and to correct for multiple hypothesis testing. To identify and replicate genes, an FDR $<0.1$ was used in the hypothesis-free discovery analyses. For replication, a gene was considered "replicated" if any of the probes showed differential expression between never and current smokers in the replication cohort at a nominal $\mathrm{P}<0.05$ and with the same direction of effect as in the discovery cohort.

Weighted gene co-expression network analysis (WGCNA). The R package WGCNA ${ }^{48}$ was used to construct gene co-expression modules with genes that are highly correlated with each other. The workflow of WGCNA starts with creating a matrix of Pearson correlations between genes, and transforming these into an adjacency matrix through soft thresholding and then converting them into a topological overlap matrix (TOM $)^{52}$. Modules are defined as groups of highly interconnected genes using the average linkage hierarchical clustering to group genes based on the topological overlap of their connectivity, followed by a dynamic cut-tree algorithm to cluster dendrogram branches into gene modules ${ }^{53}$. Each gene within a module will be assigned a Module Membership (MM) whose value ranges between 0 and 1 by relating the gene's expression profile with the module eigengene determined by the first principal component of the gene expression profiles in that module. Genes with the highest MM are considered "hub" genes, which are thought to be central to the module.

Since the discovery set and the replication set were generated using different microarray platforms, we first

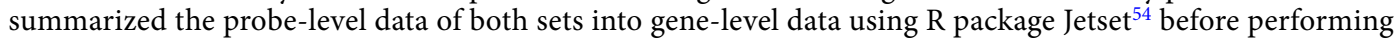
WGCNA. Using the 12,537 genes of the discovery set, we identified 37 gene modules and each module was assigned a module number. The assigned module number increases as the size of the module decreases with module 01 holding the largest number of genes. For each of the gene module, an eigengene, which is representative of the module expression profile, was obtained. Linear regression was used to identify module eigengenes that were associated with smoking status, sex, and sex-by-smoking interaction, and COPD status adjusting for ethnicity and pack-years of smoking. To replicate our findings for modules constructed in the discovery set, we calculated their eigengenes in the replication set and then tested for their relationships to smoking status, sex, and sex-by-smoking interaction using linear regression. Similar to the discovery set, the models were adjusted for potential covariates such as pack-years. FDR $<0.1$ was used as the threshold for discovery analyses and $\mathrm{P}<0.05$ was used as the threshold for replication analyses.

Gene set variation analysis (GSVA). In addition to the gene- and network-level differential expression analyses, we also performed GSVA ${ }^{55}$ to further replicate our identified gene signatures in the replication cohort. Given a gene set (a list of genes), GSVA calculates a per-subject score for the gene set using a non-parametric unsupervised method and therefore, transforming the data from a gene-by-subject matrix to a gene set-by-subject matrix. Then, differential expression analysis can be performed on the gene set instead of each individual gene to identify the association between the gene set and phenotypes. Linear regression was performed similarly to identify gene sets that were associated with sex-by-smoking interaction. $\mathrm{P}<0.05$ was used as the threshold for replication of the gene sets. The GSVA analysis was performed using R package GSVA.

Enrichment analysis. For gene modules that were successfully replicated in the replication set, we performed enrichment analyses using R package clusterProfiler ${ }^{56}$ and TFEA.ChIP ${ }^{57}$ to identify Gene Ontology (GO) biological processes and transcription factor binding sites that were enriched in the module genes.

Received: 14 January 2019; Accepted: 1 November 2019;

Published online: 26 November 2019

\section{References}

1. Vogelmeier, C. F. et al. Global Strategy for the Diagnosis, Management, and Prevention of Chronic Obstructive Lung Disease 2017 Report: GOLD Executive Summary. European Respiratory Journal 49, 1700214, https://doi.org/10.1183/13993003.00214-2017 (2017).

2. Lozano, R. et al. Global and regional mortality from 235 causes of death for 20 age groups in 1990 and 2010 : a systematic analysis for the Global Burden of Disease Study 2010. Lancet 380, 2095-2128, https://doi.org/10.1016/S0140-6736(12)61728-0 (2012).

3. Tager, I. B., Segal, M. R., Speizer, F. E. \& Weiss, S. T. The natural history of forced expiratory volumes. Effect of cigarette smoking and respiratory symptoms. The American review of respiratory disease 138, 837-849, https://doi.org/10.1164/ajrccm/138.4.837 (1988).

4. Evans, J., Chen, Y., Camp, P. G., Bowie, D. M. \& McRae, L. Estimating the prevalence of COPD in Canada: Reported diagnosis versus measured airflow obstruction. Health reports 25, 3-11 (2014). 
5. Gan, W. Q., Man, S. F., Postma, D. S., Camp, P. \& Sin, D. D. Female smokers beyond the perimenopausal period are at increased risk of chronic obstructive pulmonary disease: a systematic review and meta-analysis. Respiratory research 7,52 , https://doi. org/10.1186/1465-9921-7-52 (2006).

6. Amaral, A. F. S., Strachan, D. P., Burney, P. G. J. \& Jarvis, D. L. Female Smokers Are at Greater Risk of Airflow Obstruction Than Male Smokers. UK Biobank. American journal of respiratory and critical care medicine 195, 1226-1235, https://doi.org/10.1164/ rccm.201608-1545OC (2017).

7. Celli, B. et al. Sex differences in mortality and clinical expressions of patients with chronic obstructive pulmonary disease. The TORCH experience. American journal of respiratory and critical care medicine 183, 317-322, https://doi.org/10.1164/rccm.2010040665OC (2011).

8. Dransfield, M. T. et al. Gender differences in the severity of CT emphysema in COPD. Chest 132, 464-470, https://doi.org/10.1378/ chest.07-0863 (2007).

9. Martinez, F. J. et al. Sex differences in severe pulmonary emphysema. American journal of respiratory and critical care medicine 176, 243-252, https://doi.org/10.1164/rccm.200606-828OC (2007).

10. Tam, A. et al. Sex Differences in Airway Remodeling in a Mouse Model of Chronic Obstructive Pulmonary Disease. Am J Respir Crit Care Med 193, 825-834, https://doi.org/10.1164/rccm.201503-0487OC (2016).

11. Benowitz, N. L., Lessov-Schlaggar, C. N., Swan, G. E. \& Jacob, P. 3rd Female sex and oral contraceptive use accelerate nicotine metabolism. Clinical pharmacology and therapeutics 79, 480-488, https://doi.org/10.1016/j.clpt.2006.01.008 (2006).

12. Sin, D. D., Cohen, S. B., Day, A., Coxson, H. \& Pare, P. D. Understanding the biological differences in susceptibility to chronic obstructive pulmonary disease between men and women. Proceedings of the American Thoracic Society 4, 671-674, https://doi. org/10.1513/pats.200706-082SD (2007).

13. Van Winkle, L. S., Gunderson, A. D., Shimizu, J. A., Baker, G. L. \& Brown, C. D. Gender differences in naphthalene metabolism and naphthalene-induced acute lung injury. American journal of physiology. Lung cellular and molecular physiology 282, L1122-1134, https://doi.org/10.1152/ajplung.00309.2001 (2002).

14. Tam, A. et al. The role of female hormones on lung function in chronic lung diseases. BMC women's health 11, 24, https://doi. org/10.1186/1472-6874-11-24 (2011).

15. Tam, A., Wadsworth, S., Dorscheid, D., Man, S. F. \& Sin, D. D. Estradiol increases mucus synthesis in bronchial epithelial cells. PloS one 9 , e100633, https://doi.org/10.1371/journal.pone.0100633 (2014).

16. van den Berge, M. et al. Differential lung tissue gene expression in males and females: implications for the susceptibility to develop COPD. European Respiratory Journal, 1702567, https://doi.org/10.1183/13993003.02567-2017 (2019).

17. Obeidat, M. E. et al. The Overlap of Lung Tissue Transcriptome of Smoke Exposed Mice with Human Smoking and COPD. Scientific reports 8, 11881, https://doi.org/10.1038/s41598-018-30313-z (2018).

18. Engle, M. L. et al. Dynamic changes in lung responses after single and repeated exposures to cigarette smoke in mice. PloS one 14, e0212866, https://doi.org/10.1371/journal.pone.0212866 (2019).

19. Mele, M. et al. Human genomics. The human transcriptome across tissues and individuals. Science 348, 660-665, https://doi. org/10.1126/science.aaa0355 (2015).

20. Chen, C.-Y. et al. Sexual dimorphism in gene expression and regulatory networks across human tissues. bioRxiv, https://doi. org/10.1101/082289 (2016)

21. Vawter, M. P. et al. Gender-Specific Gene Expression in Post-Mortem Human Brain: Localization to Sex Chromosomes. Neuropsychopharmacology: official publication of the American College of Neuropsychopharmacology 29, 373-384, https://doi. org/10.1038/sj.npp.1300337 (2004).

22. Cheng, C. \& Kirkpatrick, M. Sex-Specific Selection and Sex-Biased Gene Expression in Humans and Flies. PLoS Genetics 12, e1006170, https://doi.org/10.1371/journal.pgen.1006170 (2016).

23. Trabzuni, D. et al. Widespread sex differences in gene expression and splicing in the adult human brain. 4, 2771, https://doi. org/10.1038/ncomms3771, https://www.nature.com/articles/ncomms3771\#supplementary-information (2013).

24. Werling, D. M., Parikshak, N. N. \& Geschwind, D. H. Gene expression in human brain implicates sexually dimorphic pathways in autism spectrum disorders. Nature Communications 7, 10717, https://doi.org/10.1038/ncomms10717 (2016).

25. Aghapour, M., Raee, P., Moghaddam, S. J., Hiemstra, P. S. \& Heijink, I. H. Airway Epithelial Barrier Dysfunction in COPD: Role of Cigarette Smoke Exposure. American journal of respiratory cell and molecular biology, https://doi.org/10.1165/rcmb.2017-0200TR (2017).

26. Mizushima, N. \& Komatsu, M. Autophagy: renovation of cells and tissues. Cell 147, 728-741, https://doi.org/10.1016/j. cell.2011.10.026 (2011)

27. Levine, B. \& Kroemer, G. Autophagy in the pathogenesis of disease. Cell 132, 27-42, https://doi.org/10.1016/j.cell.2007.12.018 (2008).

28. Chen, Z. H. et al. Egr-1 regulates autophagy in cigarette smoke-induced chronic obstructive pulmonary disease. PloS one 3, e3316, https://doi.org/10.1371/journal.pone.0003316 (2008).

29. Nyunoya, T. et al. Molecular processes that drive cigarette smoke-induced epithelial cell fate of the lung. American journal of respiratory cell and molecular biology 50, 471-482, https://doi.org/10.1165/rcmb.2013-0348TR (2014).

30. Kim, H. P. et al. Autophagic proteins regulate cigarette smoke-induced apoptosis: protective role of heme oxygenase-1. Autophagy 4, 887-895 (2008)

31. Lam, H. C. et al. Histone deacetylase 6-mediated selective autophagy regulates COPD-associated cilia dysfunction. The Journal of clinical investigation 123, 5212-5230, https://doi.org/10.1172/jci69636 (2013).

32. Vij, N., Chandramani-Shivalingappa, P., Van Westphal, C., Hole, R. \& Bodas, M. Cigarette smoke-induced autophagy impairment accelerates lung aging, COPD-emphysema exacerbations and pathogenesis. American journal of physiology. Cell physiology 314, C73-c87, https://doi.org/10.1152/ajpcell.00110.2016 (2018).

33. Monick, M. M. et al. Identification of an autophagy defect in smokers' alveolar macrophages. J Immunol 185, 5425-5435, https://doi. org/10.4049/jimmunol.1001603 (2010).

34. Oliván et al. Sex Differences in Constitutive Autophagy. BioMed Research International 2014, 5, https://doi.org/10.1155/2014/652817 (2014).

35. Tower, J. Mitochondrial maintenance failure in aging and role of sexual dimorphism. Archives of biochemistry and biophysics 576, 17-31, https://doi.org/10.1016/j.abb.2014.10.008 (2015).

36. Feng, Y., Yao, Z. \& Klionsky, D. J. How to control self-digestion: transcriptional, post-transcriptional, and post-translational regulation of autophagy. Trends in cell biology 25, 354-363, https://doi.org/10.1016/j.tcb.2015.02.002 (2015).

37. He, C. \& Klionsky, D. J. Regulation mechanisms and signaling pathways of autophagy. Annual review of genetics 43, 67-93, https:// doi.org/10.1146/annurev-genet-102808-114910 (2009).

38. Ivashkiv, L. B. \& Donlin, L. T. Regulation of type I interferon responses. Nat Rev Immunol 14, 36-49, https://doi.org/10.1038/nri3581 (2014).

39. Chin, K. C. \& Cresswell, P. Viperin (cig5), an IFN-inducible antiviral protein directly induced by human cytomegalovirus. Proceedings of the National Academy of Sciences of the United States of America 98, 15125-15130, https://doi.org/10.1073/ pnas.011593298 (2001).

40. Terenzi, F., Hui, D. J., Merrick, W. C. \& Sen, G. C. Distinct induction patterns and functions of two closely related interferoninducible human genes, ISG54 and ISG56. J Biol Chem 281, 34064-34071, https://doi.org/10.1074/jbc.M605771200 (2006). 
41. Nasr, N. et al. HIV-1 infection of human macrophages directly induces viperin which inhibits viral production. Blood 120, 778-788, https://doi.org/10.1182/blood-2012-01-407395 (2012).

42. Bauer, C. M. T. et al. Cigarette Smoke Suppresses Type I Interferon-Mediated Antiviral Immunity in Lung Fibroblast and Epithelial Cells. Journal of Interferon \& Cytokine Research 28, 167-179, https://doi.org/10.1089/jir.2007.0054 (2008).

43. Bauer, C. M. T., Morissette, M. C. \& Stampfli, M. R. The influence of cigarette smoking on viral infections: translating bench science to impact COPD pathogenesis and acute exacerbations of COPD clinically. Chest 143, 196-206, https://doi.org/10.1378/ chest.12-0930 (2013).

44. Ghosh, S. \& Klein, R. S. Sex Drives Dimorphic Immune Responses to Viral Infections. J Immunol 198, 1782-1790, https://doi. org/10.4049/jimmunol.1601166 (2017).

45. Klein, S. L. \& Flanagan, K. L. Sex differences in immune responses. Nature reviews. Immunology 16, 626-638, https://doi. org $/ 10.1038 /$ nri.2016.90 (2016)

46. Klein, S. L. Sex influences immune responses to viruses, and efficacy of prophylaxis and treatments for viral diseases. BioEssays: news and reviews in molecular, cellular and developmental biology 34, 1050-1059, https://doi.org/10.1002/bies.201200099 (2012).

47. McCombe, P. A., Greer, J. M. \& Mackay, I. R. Sexual dimorphism in autoimmune disease. Curr Mol Med 9, 1058-1079 (2009).

48. Obeidat, M. E. et al. Network-based analysis reveals novel gene signatures in peripheral blood of patients with chronic obstructive pulmonary disease. Respiratory research 18, 72, https://doi.org/10.1186/s12931-017-0558-1 (2017).

49. Oshiumi, H. et al. DDX60 Is Involved in RIG-I-Dependent and Independent Antiviral Responses, and Its Function Is Attenuated by Virus-Induced EGFR Activation. Cell Rep 11, 1193-1207, https://doi.org/10.1016/j.celrep.2015.04.047 (2015).

50. Obeidat, M. E. et al. The Effect of Different Case Definitions of Current Smoking on the Discovery of Smoking-Related Blood Gene Expression Signatures in Chronic Obstructive Pulmonary Disease. Nicotine \& Tobacco Research 18, 1903-1909, https://doi. org/10.1093/ntr/ntw129 (2016).

51. Ritchie, M. E. et al. limma powers differential expression analyses for RNA-sequencing and microarray studies. Nucleic acids research 43, e47, https://doi.org/10.1093/nar/gkv007 (2015).

52. Yip, A. \& Horvath, S. Gene network interconnectedness and the generalized topological overlap measure. BMC bioinformatics 8, 22 (2007).

53. Langfelder, P., Zhang, B. \& Horvath, S. Defining clusters from a hierarchical cluster tree: the Dynamic Tree Cut package for R. Bioinformatics 24, 719-720, https://doi.org/10.1093/bioinformatics/btm563 (2008).

54. Li, Q., Birkbak, N. J., Gyorffy, B., Szallasi, Z. \& Eklund, A. C. Jetset: selecting the optimal microarray probe set to represent a gene. BMC Bioinformatics 12, 474, https://doi.org/10.1186/1471-2105-12-474 (2011).

55. Hänzelmann, S., Castelo, R. \& Guinney, J. GSVA: gene set variation analysis for microarray and RNA-Seq data. BMC Bioinformatics 14, 7, https://doi.org/10.1186/1471-2105-14-7 (2013).

56. Yu, G., Wang, L. G., Han, Y. \& He, Q. Y. clusterProfiler: an R package for comparing biological themes among gene clusters. Omics: a journal of integrative biology 16, 284-287, https://doi.org/10.1089/omi.2011.0118 (2012).

57. Puente-Santamaria, L. \& del Peso, L. TFEA.ChIP: A tool kit for transcription factor binding site enrichment analysis capitalizing on ChIP-seq datasets. bioRxiv, 303651, https://doi.org/10.1101/303651 (2018).

\section{Acknowledgements}

M.O. is a fellow of the Parker B. Francis Foundation and a Scholar of the Michael Smith Foundation for Health Research. D.D.S. holds Canada Research Chair in COPD.

\section{Author contributions}

Conceived and designed the study: M.O., T.L.H., J.L., D.D.S. Gene expression data analyses: M.O., C.X.Y., H.S., I.D., C.W.T.Y., E.K.H.K., Wrote the manuscript: M.O., D.D.S., J.L., S.M, A.I.H.C. Discussed results and implications and commented on the manuscript at all stages: all co-authors. All authors read and approved the final manuscript.

\section{Competing interests}

The authors declare no competing interests.

\section{Additional information}

Supplementary information is available for this paper at https://doi.org/10.1038/s41598-019-54051-y.

Correspondence and requests for materials should be addressed to M.O.

Reprints and permissions information is available at www.nature.com/reprints.

Publisher's note Springer Nature remains neutral with regard to jurisdictional claims in published maps and institutional affiliations.

Open Access This article is licensed under a Creative Commons Attribution 4.0 International License, which permits use, sharing, adaptation, distribution and reproduction in any medium or format, as long as you give appropriate credit to the original author(s) and the source, provide a link to the Creative Commons license, and indicate if changes were made. The images or other third party material in this article are included in the article's Creative Commons license, unless indicated otherwise in a credit line to the material. If material is not included in the article's Creative Commons license and your intended use is not permitted by statutory regulation or exceeds the permitted use, you will need to obtain permission directly from the copyright holder. To view a copy of this license, visit http://creativecommons.org/licenses/by/4.0/.

(C) The Author(s) 2019 